\title{
A Unified Orbital Model of Delocalised and Localised Currents in Monocycles, from Annulenes to Azabora-heterocycles
}

\author{
Alessandro Soncini, ${ }^{[a]}$ Carmen Domene ${ }^{[b]}$ Jeroen J. Engelberts, ${ }^{[c]}$ Patrick W. Fowler, ${ }^{[a]}$ \\ André Rassat, ${ }^{*[d]}$ Joop H. van Lenthe, ${ }^{[c]}$ Remco W. A. Havenith, ${ }^{[c]}$ and \\ Leonardus W. Jenneskens ${ }^{[\mathrm{e}]}$
}

\begin{abstract}
Why are some $(4 n+2) \pi$ systems aromatic, and some not? The ipsocentric approach to the calculation of the current density induced in a molecule by an external magnetic field predicts a four-electron diatropic (aromatic) ring current for $(4 n+2) \pi$ carbocycles and a two-electron paratropic (antiaromatic) current for $(4 n) \pi$ carbocycles. With the inclusion of an electro-
\end{abstract}

negativity parameter, an ipsocentric frontier-orbital model also predicts the transition from delocalised currents in carbocycles to nitrogen-localised currents in alternating azabora-heterocy-

Keywords: aromaticity • boron • nitrogen heterocycles $\cdot$ ring current • valence bond cles, which rationalises the differences in (magnetic) aromaticity between these isoelectronic $\pi$-conjugated systems. Ab initio valence-bond calculations confirm the localisation predicted by the naive model, and coupled-Hartree-Fock calculations give currentdensity maps that exhibit the predicted delocalised-to-localised/carbocycle-heterocycle transition.

\section{Introduction}

Planar conjugated carbocyclic compounds show a strong link between $\pi$-electron count and molecular properties. Hückel's long-standing $4 n+2$ rule distinguishes aromatic from antiaromatic monocycles, and correlates the energetics

[a] Dr. A. Soncini, Prof. P. W. Fowler

Department of Chemistry, University of Exeter

Stocker Road, Exeter EX4 4QD (UK)

Fax: (+44)1392-263-434

E-mail: a.soncini@ex.ac.uk

$$
\text { p.w.fowler@ex.ac.uk }
$$

[b] Dr. C. Domene

Physical and Theoretical Chemistry Laboratory, Oxford University South Parks Road, Oxford OX1 3QZ (UK)

[c] J. J. Engelberts, Prof. J. H. van Lenthe, Dr. R. W. A. Havenith Debye Institute, Theoretical Chemistry Group, Utrecht University Padualaan 8, 3584 Utrecht (The Netherlands)

[d] Prof. A. Rassat

École Normale Supérieure, Département de Chimie, UMR 8640 CNRS-ENS-Université Pierre-et-Marie-Curie

24 rue Lhomond, 75231 Paris CEDEX 05 (France)

Fax: (+33) 144-323-325

E-mail: andre.rassat@ens.fr

[e] Prof. L. W. Jenneskens

Debye Institute, Department of Physical Organic Chemistry Utrecht University

Padualaan 8, 3584 Utrecht (The Netherlands) of these systems with their characteristic magnetic properties. $^{[1]}$ However, the periodic table offers wide possibilities for the construction of isoelectronic heterocycles, and for many of them, direct transferability of the implied relationship between aromaticity and electron count is questionable or even counterfactual. The replacement of $\mathrm{CC}$ by $\mathrm{BN}$, for example, is known to lead to marked differences in magnetic properties: ${ }^{[2]}$ the strong ring currents of the carbocycle vanish in the isoelectronic azabora-heterocycles, $\mathrm{B}_{N / 2} \mathrm{~N}_{N / 2} \mathrm{H}_{N}$ (1-3). Herein we show that a simple frontier-orbital model<smiles>B1=NB1</smiles><smiles>b1bncbn1</smiles><smiles>B1=NB=NBNBN1</smiles><smiles>B1BN1</smiles><smiles>B1NBNBN1</smiles><smiles>B1NBNBNBN1</smiles>

1
3 
of induced currents can in fact cope with both extremes, predicting the transition from delocalised to localised magnetic behaviour that is found in full ab initio current-density maps.

The ipsocentric model ${ }^{[3-5]}$ gives an accurate account of $\pi$ ring currents in planar carbocycles in terms of frontier-orbital contributions. The hallmarks of aromaticity and antiaromaticity on the magnetic criterion, ${ }^{[6-9]}$ that is, the diatropic currents of $4 n+2$ systems and the paratropic currents ${ }^{[10]}$ of planar $4 n$ systems are attributed in this model to four and two frontier electrons, respectively. ${ }^{[4]}$ In both cases, the sense of the ring current follows directly from the symmetry properties of the HOMO-LUMO transition, and the predictions of a simple orbital theory are borne out in full $a b$ initio calculations of current-density maps. ${ }^{[3,4]}$ For the $4 n+2 \pi$ heterocycles, borazine and boroxine, however, ab initio maps show only localised circulation of the $\pi$ electrons around the electronegative nuclei. ${ }^{[2]}$ How does the simple orbital model deal with this dichotomy? In particular, why does it not predict ring currents for all planar $4 n$ and $4 n+2$ $\pi$ systems? It is shown here that a generalisation of the pictorial orbital model is able to account for both the presence of global currents in carbocycles, and their absence in boron-nitrogen analogues such as borazine (2) and the eight-membered borazocine (3).

\section{Results and Discussion}

The aromaticity analogy: The considerations of magnetic aromaticity have their counterparts in the chemistry of these boron-nitrogen systems. Although $6 \pi$-electron borazine (2) was originally called "an inorganic benzene" $"[11]$ and believed to have a resonance energy similar to that of benzene, it was soon recognised that it took part in few reactions typical of aromatic systems. For instance, borazine gives a tris-adduct with hydrogen chloride. ${ }^{[12]}$ It is now generally agreed that borazine is not aromatic. In contrast to benzene, the $\pi$ electrons are localised on the more negative nitrogen atoms, even if some chemical behaviour is reminiscent of that of benzene (e.g. the formation of a weakly puckered hexaethylborazine tricarbonyl chromium complex). ${ }^{[13]}$

The parent compounds $\mathrm{B}_{N / 2} \mathrm{~N}_{N / 2} \mathrm{H}_{N}$ with $N \neq 6$ have not been synthesised, although many derivatives of the homologues diazadiboretidine (also known as diazadiboretane and diazadiborete) (1) and borazocine (tetrazaborocine, tetrazaborocane) (3) have been prepared. ${ }^{[14]}$ Interconversions between them (and with borazine) are possible, and their chemical properties differ strongly from those of the corresponding $4 \pi$ and $8 \pi$ carbocycles. For instance, the four-membered ring survives thermal elimination of isobutene from a tert-butyl derivative. ${ }^{[14 b]}$ Thus, in spite of their $4 n \pi$ electrons, these systems do not show the reactivity expected of an antiaromatic molecule. This again can be attributed to localisation of the $\pi$ electrons onto the more electronegative nitrogen atoms. Similarly, energy calculations ${ }^{[11 b, 15]}$ suggest that $\mathbf{1}$ is less antiaromatic than cyclobutadiene, in agreement with the Hückel prediction that the antiaromaticity in $\mathrm{X}_{2} \mathrm{Y}_{2} \mathrm{H}_{4}$ type four-membered rings decreases with increasing electronegativity difference between $\mathrm{X}$ and $\mathrm{Y} \cdot{ }^{[16]}$ By using the ipsocentric model, we show here that diazadiboretidine (1), borazine (2) and borazocine (3) are in fact all nonaromatic.

Aromaticity, ring currents and frontier orbitals: If aromaticity is to be defined by magnetic criteria ${ }^{[9]}$ what is needed for its theoretical characterisation is a reliable account of the currents induced by an external magnetic field since susceptibility, nuclear shielding and other response properties are all integrals of this current density. Realistic current-density maps can be obtained by a computationally efficient procedure, the ipsocentric method, which avoids the gauge-dependence problem by taking each point in space as the origin of the vector potential generating the magnetic field. ${ }^{[17,18]}$ This choice has the important conceptual advantage of yielding a unique decomposition into nonredundant orbital contributions. ${ }^{[3]}$ The total current density is obtained as the sum over transitions from occupied to unoccupied orbitals, and is governed by symmetry rules, and modulated by energy denominators.

For a planar molecule in a perpendicular magnetic field, the symmetries determining the sense of circulation of the induced current about the molecular origin are those of inplane translations $\left(T_{x}, T_{y}\right)$ and the in-plane rotation $\left(R_{z}\right)$. A simple but powerful symmetry rule can be deduced. If a product of occupied and unoccupied orbitals contains the $T_{x}$ or $T_{y}$ symmetry, but not the $R_{z}$ symmetry, the contribution of the transition to the current has the diatropic sense, and if it contains the $R_{z}$ symmetry, but not the $T_{x}$ or $T_{y}$ symmetry, the contribution has the reverse paratropic sense. If neither symmetry is present, the transition is inactive; if both, more detailed analysis is needed ${ }^{[3,19]}$ In terms of the magnetic criterion, the resultant of all such contributions determines the aromaticity or antiaromaticity (net diatropicity or paratropicity of the ring current) of a monocycle. As the contributions are weighted by orbital energy differences, frontier orbitals generally dominate.

Currents in carbocycles: Current-density maps from ab initio calculations ${ }^{[4]}$ on the archetypal carbocycles, benzene and (planarised ${ }^{[20]}$ ) cyclooctatetraene (COT), are shown in Figure 1. For each molecule, the maps show total $\pi$ and $\sigma$ contributions to the induced current density. As expected, the currents arising from the $\pi$ electrons are, respectively, strongly diatropic in benzene and strongly paratropic in COT. The currents are dominated by HOMO contributions in both cases.

An angular-momentum analysis shows how the symmetry rules account for these features. The Hückel $\pi$ molecular orbitals of a cycle of $N$ identical atoms in full $D_{N h}$ symmetry have well-defined angular-momentum properties with respect to the principal axis. At each successive energy level, the quantum number, $\lambda(=0,1 \ldots, N / 2)$, increases by one. In a cycle with $N=4 n+2$ electrons, the HOMO and LUMO correspond to $\lambda=n$ and $n+1$, respectively, whereas for a cycle 

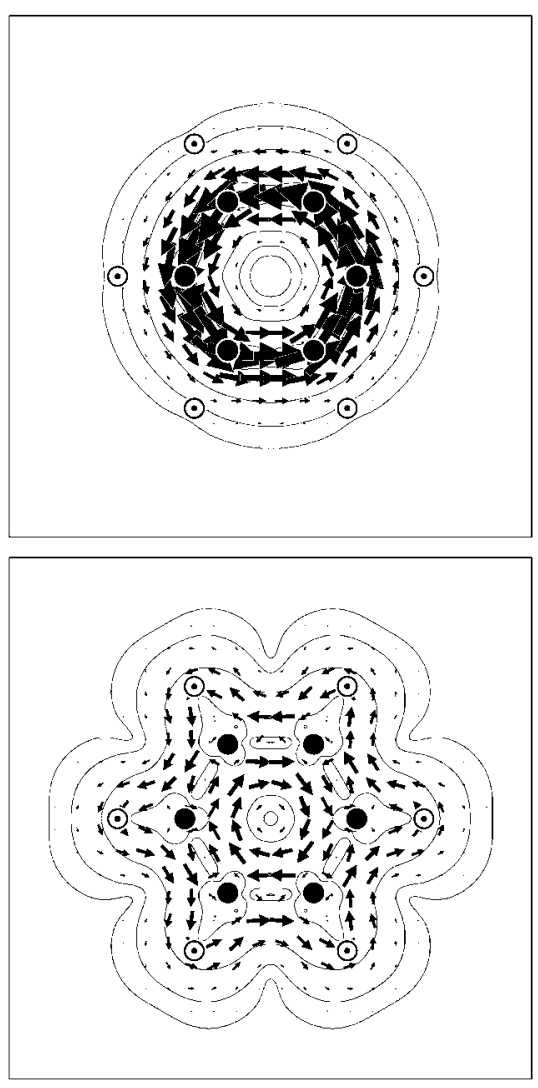

Figure 1. Maps of the current density induced in benzene (left) and (planar) cyclooctatetraene (right) by a perpendicular external magnetic field. Contributions of the $\pi$ (top) and $\sigma$ orbitals (bottom) calculated by the RHF/6-31G** ipsocentric approach are plotted at one bohr above the molecular plane. Anticlockwise circulations are diatropic, clockwise circulations paratropic. $\bigcirc:$ Carbon atoms; $\odot$ : hydrogen atoms.

LUMO and HOMO-LUMO+1 transitions to diatropic contributions. Here, the smaller HOMO-LUMO splitting ensures dominance of the paratropic current. Decomposition of the carbocycle ab initio $\pi$ maps into orbital contributions confirms the above analysis. ${ }^{[4]}$

A model for currents in azabora-heterocycles: To adapt the pictorial molecular-orbital analysis to the ipsocentric model for currents in alternating heterocycles such as borazine (2) and homologues $\mathbf{1}$ and $\mathbf{3}$, it is sufficient to include one extra feature. To deal with heteroatoms in Hückel theory it is necessary to modify the Coulomb and/or resonance integral parameters: for boron and nitrogen (as zero- and two-electron donors), the recommended parameters are $\alpha_{\mathrm{B}}=\alpha-\beta$ and $\alpha_{\mathrm{N}}=$ $\alpha+1.5 \beta .^{[22]}$ Hückel calculations using $\alpha_{\mathrm{B}}=\alpha-1.1 \beta$ and $\alpha_{\mathrm{N}}=$ $\alpha+1.5 \beta$ have been reported for 1-3. ${ }^{[16]}$ In the simplest model of an equilateral $\mathrm{B}_{N / 2} \mathrm{~N}_{N / 2} \mathrm{H}_{N}$ cycle that allows for the differing

with $N=4 n$ electrons, in the closed-shell configuration obtained on distortion to $D_{(N / 2) h}$ symmetry, the HOMO and LUMO are derived from the splitting of a pair of originally degenerate orbitals with $\lambda=n$.

The canonical molecular orbitals are the delocalised set $\left\{\psi_{\lambda, \mathrm{c} / \mathrm{s}}\right\}$, which produce uniform $\pi$-charge distribution on all the carbon atoms. Functions $\psi_{\lambda, \mathrm{c}}$ and $\psi_{\lambda, \mathrm{s}}$ (degeneracy $d_{\lambda}$ ) have coefficients on centre $r$ equal to $\sqrt{d_{\lambda} / N} \cos (2 \pi \lambda r / N)$ and $\sqrt{d_{\lambda} / N} \sin (2 \pi \lambda r / N)$, respectively. ${ }^{[21]}$ Unless $\lambda=0$ (or $N / 2$, if allowed), the two functions share a single, well-defined angular momentum and are degenerate. For $\lambda=0$ and $N / 2$, the sine partner vanishes identically. For other values of $\lambda$ the sine/cosine form is simply a choice that gives convenient nodal properties.

When orbitals have well-defined values of $\lambda$, the symmetry selection rules for occupied-to-unoccupied transitions are reduced to the following: ${ }^{[4]}$ a diatropic contribution arises from a transition in which $\Delta \lambda=+1$ and a paratropic contribution arises from a transition in which $\Delta \lambda=0$. Thus, for a $4 n+2$ cycle with maximum symmetry, only the HOMO-LUMO transition is active, and is responsible for the entire (four-electron) diatropic current. For a $4 n$ cycle with the lower symmetry of the closed shell, two types of transition are active: the HOMO-LUMO transition leads to a two-electron paratropic contribution, and HOMO-1- electronegativities of boron and nitrogen, symmetrical changes are made to the Coulomb parameters, that is, $\alpha_{\mathrm{B}}=$ $\alpha-\eta \beta$ and $\alpha_{\mathrm{N}}=\alpha+\eta \beta$, where $\eta$ is positive. Variation of the dimensionless quantity $\eta$ from 0 to $\approx 1$ thus gives a oneparameter model to correlate the properties of annulenes and BN cycles.

The implications of this model for six- and eight-membered cycles, as representatives of $4 n+2$ and $4 n \pi$ systems, respectively, are now investigated in detail. Direct solution of the Hückel problem with modified $\alpha$ values gives energy levels $\{\varepsilon\}$ and molecular orbitals $\{\phi\}$ from which the consequences for ring currents can be deduced. In the limit, $\eta=0$, the canonical molecular orbitals are $\left\{\psi_{\lambda, \mathrm{c} / \mathrm{s}}\right\}$; when $\eta \neq 0$, they are linear combinations of this starting set.

Currents in the six-membered cycle: The secular equations for the alternating six-membered cycle have maximal $D_{3 h}$ $(\eta \neq 0)$ or $D_{6 h}(\eta=0)$ symmetry. The energies are $\left(D_{3 h} / D_{6 h}\right.$ labels, $d_{\lambda}=$ degeneracy):

$$
\begin{aligned}
& \varepsilon_{0}=\alpha+\sqrt{4+\eta^{2}} \beta\left(\mathrm{A}_{2}^{\prime \prime} / \mathrm{A}_{2 \mathrm{u}}\right), d_{\lambda}=1 \\
& \varepsilon_{1}=\alpha+\sqrt{1+\eta^{2}} \beta\left(\mathrm{E}^{\prime \prime} / \mathrm{E}_{1 \mathrm{~g}}\right), d_{\lambda}=2
\end{aligned}
$$




$$
\begin{aligned}
& \varepsilon_{2}=\alpha-\sqrt{1+\eta^{2}} \beta\left(\mathrm{E}^{\prime \prime} / \mathrm{E}_{2 \mathrm{u}}\right), d_{\lambda}=2 ; \\
& \varepsilon_{3}=\alpha-\sqrt{4+\eta^{2}} \beta\left(\mathrm{A}_{2}^{\prime \prime} / \mathrm{B}_{2 \mathrm{~g}}\right), d_{\lambda}=1 ;
\end{aligned}
$$

When $\eta$ is nonzero, functions with different $\lambda$ are allowed to mix according to their symmetries in the lower $D_{3 h}$ group, that is, in $\mathrm{A}_{2}^{\|}, \lambda=0$ mixes with $\lambda=3$, and in $E^{\|}, \lambda=1$ mixes with $\lambda=2$ (retaining the sine/cosine distinction). As $\eta$ increases, the degree of mixing increases, and is described by angles $\mu$ and $\nu$, where $\eta=2 \tan 2 \mu=\tan 2 v$ :

$$
\begin{aligned}
& \phi_{0, \mathrm{c}}=\cos \mu \psi_{0, \mathrm{c}}-\sin \mu \psi_{3, \mathrm{c}}, \\
& \phi_{1, \mathrm{c}}=\cos v \psi_{1, \mathrm{c}}-\sin v \psi_{2, \mathrm{c}}, \phi_{1, \mathrm{~s}}=\cos v \psi_{1, \mathrm{~s}}+\sin v \psi_{2, \mathrm{~s}}, \\
& \phi_{2, \mathrm{c}}=\sin v \psi_{1, \mathrm{c}}+\cos v \psi_{2, \mathrm{c}}, \phi_{2, \mathrm{~s}}=-\sin v \psi_{1, \mathrm{~s}}+\cos v \psi_{2, \mathrm{~s}}, \\
& \phi_{3, \mathrm{c}}=\sin \mu \psi_{0, \mathrm{c}}+\cos \mu \psi_{3, \mathrm{c}} .
\end{aligned}
$$

When $\eta=0, \mu$ and $v$ are zero; in the limit of a large $\eta$, the molecular orbitals of the alternating six-membered cycle become exact 50:50 mixtures, with $|\mu|=|v|=45^{\circ}$. When $\eta=$ $1, \phi_{1, \mathrm{c} / \mathrm{s}}$ still contains $95 \%$ of the benzene $\psi_{0, \mathrm{c}}\left(|\mu|=13.3^{\circ}\right)$, but the HOMO pair, $\phi_{1, \mathrm{c} / \mathrm{s}}$, contains a $15 \%$ admixture of the benzene LUMO, $\psi_{2, \mathrm{c} / \mathrm{s}}\left(|v|=22.5^{\circ}\right)$. Figure 2 a shows the correlation of orbitals and energies with $\eta$ for the six-membered cycle. Hückel $\pi$ charges reflect this shift with respect to the uniform charge distribution of benzene, with $1 \pm$ $1 / 2\left(\frac{1}{\sqrt{5}}+\sqrt{2}\right) \approx 1.62$ and $0.38 \pi$ electrons on the nitrogen and boron atoms when $\eta=1$. As $\eta$ increases, bonding orbitals concentrate on the electronegative nitrogen atoms, and antibonding orbitals on the electropositive boron atoms, the functions becoming more "localised".

The mixing has crucial consequences for the predicted currents. When $\eta=0$, the dominant HOMO-LUMO transition responsible for the ring current of the carbocycle is from $\left\{\psi_{1, \mathrm{c}}, \psi_{1, \mathrm{~s}}\right\}$ to $\left\{\psi_{2, \mathrm{c}}, \psi_{2, \mathrm{~s}}\right\}$ for which $\Delta \lambda=1$; the transition is therefore purely translational and contributes only diatropic current. For all $\eta>0$, as HOMO and LUMO each contain $\lambda=1$ and 2 contributions, the transition includes $\Delta \lambda=1$ and 0 components, and the current gains partial paratropic character. The global diatropic current of benzene therefore weakens as $\eta$ increases.

A first interpretation of the effect of progressive orbital mixing on the ring current in a $4 n+2$ cycle can be found by using the simplest approach, the Hückel-London model. ${ }^{[6]}$ In this model, as formulated by McWeeny, ${ }^{[23]}$ the ring current per unit area of a cycle is proportional to the reduced bond current, $J_{r s}$, where $p_{r s}$ is the $\pi$ bond-order between adjacent atoms $r$ and $s$ and $\bar{\pi}_{r s, r s}$ is the imaginary bond-bond polarisability. ${ }^{[1]}$

$J_{r s}=\left(p_{r s}+\beta \bar{\pi}_{r s, r s}\right) \beta$

In a monocycle, $J, p$ and $\bar{\pi}$ are independent of the choice of adjacent pair. For benzene, $p=2 / 3$ and $\pi=-5 / 9 \beta^{-1}$. For borazine with $\eta=1$, a straightforward calculation gives $p=$
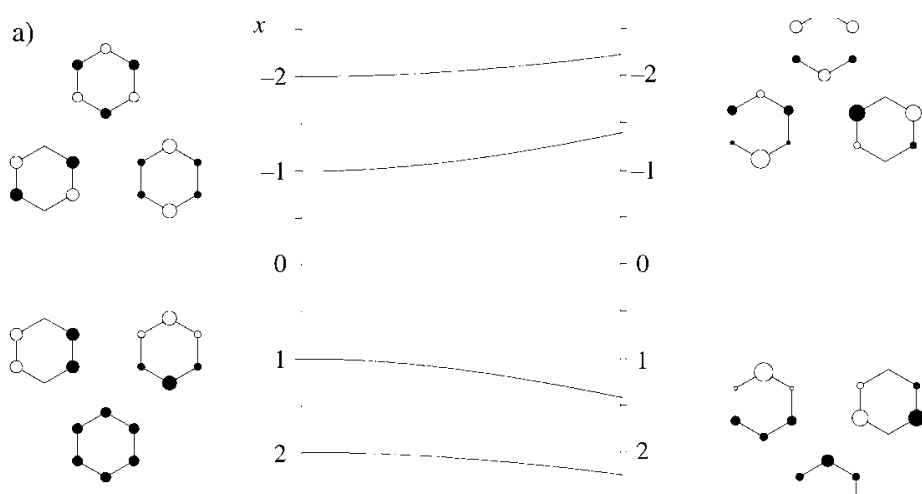

0
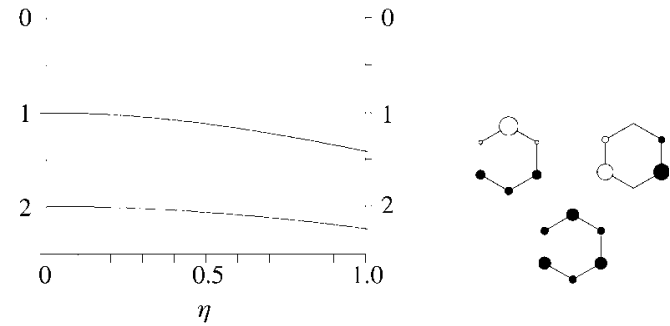

b)
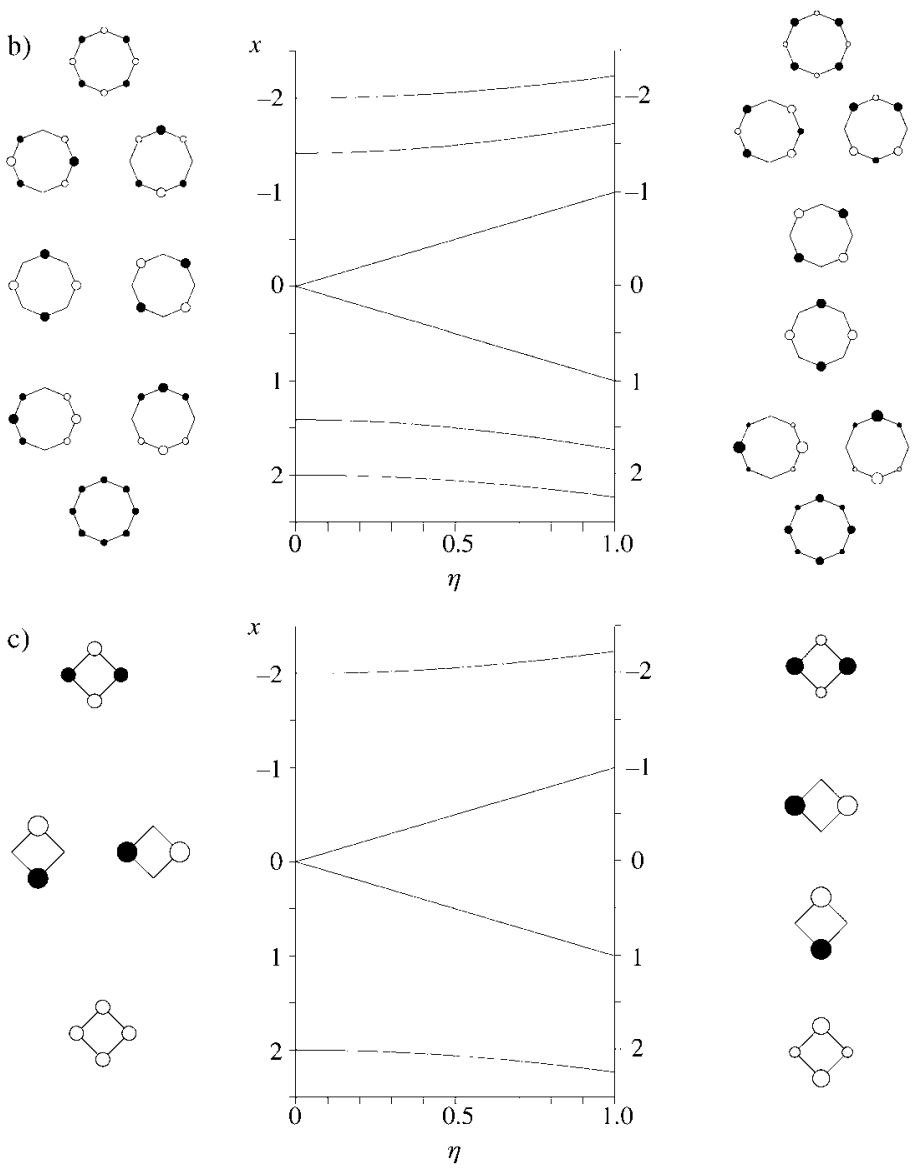

Figure 2. Correlation diagrams of energy and orbital composition in a Hückel model of a) $\mathrm{B}_{3} \mathrm{~N}_{3} \mathrm{H}_{6}$, b) $\mathrm{B}_{4} \mathrm{~N}_{4} \mathrm{H}_{8}$ and c) $\mathrm{B}_{2} \mathrm{~N}_{2} \mathrm{H}_{4}$, as modelled by the single parameter $\eta$, where $\alpha_{\mathrm{B}}=\alpha-\eta \beta$ and $\alpha_{\mathrm{N}}=\alpha+\eta \beta$. The graphs show the variation of orbital energy, $x=(\varepsilon-\alpha) / \beta$, with $\eta$.

$1 / 3\left(\frac{2}{\sqrt{5}}+\frac{1}{\sqrt{2}}\right)$ and $\bar{\pi}=-1 / 9\left(\sqrt{5}+\frac{13}{4 \sqrt{2}}\right) \beta^{-1}$, from which the borazine:benzene ratio of ring-current intensity, assuming an equal ring area, is $\left(\frac{1}{\sqrt{5}}-\frac{1}{4 \sqrt{2}}\right) \approx 0.27$. This ratio tends to zero as the electronegativity difference parameter $\eta$ tends to infinity. Thus, this crude model, in which current is constrained to flow along the straight lines between adjacent atoms, predicts that an increase in $\eta$ leads to diminution and eventual extinction of the global diatropic current. 
The picture can be refined by allowing for the spatial extent of the current outside the direct line of centres. The pseudo- $\pi$ model $^{[24]}$ includes explicit basis functions, and simulates $\pi$ ring currents by using a $\sigma$ model based on the exact symmetry equivalence between the $\sigma$ orbitals of cyclic $\mathrm{H}_{N}$ atoms and the $\pi$ orbitals of the $N$-membered carbocycle. It turns out that ipsocentric in-plane currents calculated for the $\mathrm{H}_{N}$ system with a minimal basis set give an excellent match to the out-of-plane currents of the carbocycle calculated with a large basis set. Figure 3 presents a pseudo- $\pi$

$$
\begin{aligned}
& \varepsilon_{1}=\alpha+\sqrt{2+\eta^{2}} \beta\left(\mathrm{E}_{\mathrm{g}} / \mathrm{E}_{1 \mathrm{~g}}\right), d_{\lambda}=2 ; \\
& \varepsilon_{2}^{ \pm}=\alpha \pm \eta \beta\left(\mathrm{B}_{1 \mathrm{u}}+\mathrm{B}_{2 \mathrm{u}} / \mathrm{E}_{2 \mathrm{u}}\right), d_{\lambda}=2 \text { at } \eta=0 ; d_{\lambda}=1+1 \text { at } \eta \neq 0 ; \\
& \varepsilon_{3}=\alpha-\sqrt{2+\eta^{2}} \beta\left(\mathrm{E}_{\mathrm{g}} / \mathrm{E}_{3 \mathrm{~g}}\right), d_{\lambda}=2 ; \\
& \varepsilon_{4}=\alpha-\sqrt{4+\eta^{2}} \beta\left(\mathrm{A}_{2 \mathrm{u}} / \mathrm{B}_{2 \mathrm{u}}\right), d_{\lambda}=1 ;
\end{aligned}
$$

In the carbocyclic system with full symmetry, the degeneracy of $\psi_{2, \mathrm{c}}$ and $\psi_{2, \mathrm{~s}}$ leads to an open-shell configuration which can be stabilised in various ways: distortion to $D_{2 d}$ leads to the tub-shaped equilibrium geometry of the free COT molecule; in-plane relaxation to $D_{4 h}$ yields a geometry similar to those found in "clamped"-substituted COT systems. ${ }^{[20]}$ In spite of its bond alternation, planar $D_{4 h}$ COT retains fully delocalised orbitals and the ring current of the equilateral carbocycle, as Figure 1 shows. ${ }^{[4,20 b]}$

In the heterocyclic system, when $\eta \neq 0$, with the nitrogen atom as atom $0, \psi_{2, c}$ as the bonding and $\psi_{2, \mathrm{~s}}$ the antibonding partner, the assignment of labels $\mathrm{B}_{1 \mathrm{u}}$ and $\mathrm{B}_{2 \mathrm{u}}$ depends on the setting of $D_{4 h}$ within $D_{8 h}$.

simulation of the heterocyclic ring in which the boron and nitrogen atoms are modelled by one-electron atoms with nuclear charges of $+0.7 e$ and $+1.3 e$, each carrying STO-3G s and $\mathrm{p}$ functions (exponent $1 \mathrm{a}_{0}^{2}$ ), the charge difference playing the role of the $\eta$ parameter in the Hückel analysis, and the in-plane $p$ functions giving radial flexibility to charge and current densities. Pure symmetry arguments predicted opposed paramagnetic and diamagnetic currents; the pseudo- $\pi$ map shows that they occupy different regions of space, with diamagnetic circulation on the outside and paramagnetic circulation on the inside of the ring, the net effect being a set of circulations centred on electronegative atoms. In the graph-theoretical Hückel-London model, this spatial pattern of opposed local currents was expressed as a reduction of the sole available parameter, the bond current. Both models therefore essentially agree on the effect of the electronegativity difference on the ring current.

Currents in the eight-membered cycle: For the alternating eight-membered cycle, the maximal symmetry is $D_{4 h}(\eta \neq 0)$ or $D_{8 h}(\eta=0)$ and the energy levels $\varepsilon_{\lambda}\left(D_{4 h} / D_{8 h}\right.$ labels, $d_{\lambda}=$ degeneracy) are:

$\varepsilon_{0}=\alpha+\sqrt{4+\eta^{2}} \beta\left(\mathrm{A}_{2 \mathrm{u}} / \mathrm{A}_{2 \mathrm{u}}\right), d_{\lambda}=1$
For a general value of $\eta$, the mixing of angular-momentum components is again described by two angles, $\mu$ and $\kappa$, with $\eta=2 \tan 2 \mu=\sqrt{2} \tan 2 \kappa$.

$$
\begin{aligned}
& \phi_{0, \mathrm{c}}=\cos \mu \psi_{0, \mathrm{c}}-\sin \mu \psi_{4, \mathrm{c}}, \\
& \phi_{1, \mathrm{c}}=\cos \kappa \psi_{1, \mathrm{c}}-\sin \kappa \psi_{3, \mathrm{c}}, \phi_{1, \mathrm{~s}}=\cos \kappa \psi_{1, \mathrm{~s}}+\sin \kappa \psi_{3, \mathrm{~s}}, \\
& \phi_{2, \mathrm{c}}=\psi_{2, \mathrm{c}}, \phi_{2, \mathrm{~s}}=\psi_{2, \mathrm{~s}}, \\
& \phi_{3, \mathrm{c}}=\sin \kappa \psi_{1, \mathrm{c}}+\cos \kappa \psi_{3, \mathrm{c}}, \phi_{3, \mathrm{~s}}=-\sin \kappa \psi_{1, \mathrm{~s}}+\cos \kappa \psi_{3, \mathrm{~s}}, \\
& \phi_{4, \mathrm{c}}=\sin \mu \psi_{0, \mathrm{c}}+\cos \mu \psi_{4, \mathrm{c}} .
\end{aligned}
$$

Note that functions $\psi_{2, \mathrm{c}}$ and $\psi_{2, \mathrm{~s}}$, as the HOMO and LUMO, remain unmixed for all values of $\eta$, separated by a gap that is linear in $\eta$, that is, $2 \eta$. Each is perfectly localised, the HOMO on the nitrogen atom and the LUMO on the boron atom. In contrast, with increasing values of $\eta$, the functions $\phi_{0, \mathrm{c}}, \phi_{1, \mathrm{c}}, \phi_{1, \mathrm{~s}}, \phi_{3, \mathrm{c}}, \phi_{3, \mathrm{~s}}$ and $\phi_{4, \mathrm{c}}$ become increasingly localised on the electronegative atoms: when $\eta=1$, the nitrogen and boron atoms have Hückel $\pi$ populations of $1 \pm$ $1 / 2\left(1 / 2+\frac{1}{\sqrt{3}}+\frac{1}{2 \sqrt{5}} \approx 1.65\right.$ and 0.35 electrons, respectively. When $\eta=1$, here representing planar $\mathrm{B}_{4} \mathrm{~N}_{4} \mathrm{H}_{8},|\mu|=13.3^{\circ}$ and $|\kappa|$ $=17.6^{\circ}$ so that $\phi_{0, \mathrm{c}}$ contains $5 \%$ of $\psi_{4, \mathrm{css}}$ and HOMO-1 $\phi_{1, \mathrm{c} / \mathrm{s}}$ contains $10 \%$ of $\psi_{3, \mathrm{c} / \mathrm{s}}$. Figure $2 \mathrm{~b}$ shows the correlation of or- 
bitals and energies with $\eta$ for the eight-membered cycle.

The symmetry argument for deducing the currents in $4 n$ systems involves several steps. Initially $(\eta \approx 0)$, the current is dominated by the HOMO-LUMO transition across a small energy gap. This $\Delta \lambda=0$ transition generates an intense, purely paratropic, ring current. As $\eta$ increases, the HOMOLUMO gap opens, and the intensity of the current falls but remains paramagnetic. Simultaneously, the separation of the HOMO -1 and the LUMO and that of the HOMO and the LUMO+1, increase, but only slowly; both correspond to $\Delta \lambda= \pm 0$ diatropic contributions to current. Thus, as $\eta$ increases, the original global paratropic (antiaromatic) ring current is subject to intrinsic reduction and increasingly significant cancellation.

In the McWeeny form of the Hückel-London theory, ${ }^{[23]}$ the ring current of the eight-membered cycle when $\eta$ is small is dominated by the HOMO-LUMO contribution to the imaginary bond-bond polarisability, which is $-1 / 16 \beta \eta$, and hence falls sharply as $\eta$ increases from the planar-constrained form of COT (where $\eta=0$ ) to the heterocycle. When $\eta=1$, the eight-membered cycle has $p=1 / 2\left(\frac{1}{\sqrt{3}}+\frac{1}{\sqrt{5}}\right)$ and $\bar{\pi}=-1 / 8\left(\frac{11}{3 \sqrt{3}}+\frac{7}{2 \sqrt{5}}+1 / 2\right) \beta^{-1}$ to give bond currents $J=$ $\frac{\sqrt{3}}{72}+\frac{\sqrt{5}}{80}-1 / 16 \approx-0.105$, which constitute a reduced but still net paratropic circulation. If the ratio of the areas of the sixand eight-membered cycles is 9:16, a ring current of about a sixth of the strength of the (diatropic) benzene current is implied. When, as for the six-membered cycles, the spatial extent of the current in the eight-membered cycle is taken into account in a pseudo- $\pi$ calculation (Figure 3 ), the Hückel-London result is seen as a simplified representation of the nitrogen-centred currents.

Currents in the four-membered cycle: For the four-membered ring, $\mathrm{B}_{2} \mathrm{~N}_{2} \mathrm{H}_{4}$, the extinction of current with increasing $\eta$ follows the same pattern as that for the eight-membered cycle. The four orbital energies are now $\varepsilon_{0}=\alpha+\sqrt{4+\eta^{2}} \beta, \varepsilon_{1}{ }^{ \pm}$ $=\alpha \pm \eta \beta, \varepsilon_{2}=\alpha-\sqrt{4+\eta^{2}} \beta$, again giving a linear HOMOLUMO gap of $2 \eta$ (see Figure $2 \mathrm{c}$ ), and yielding a paratropic current that weakens with $\eta$ and is increasingly cancelled by the diatropic current that arises from the transitions across the HOMO-LUMO+1 and HOMO-1-LUMO gaps of $\eta+\sqrt{4+\eta^{2}} \beta$. In the Hückel-London theory, when $\eta=1$ the reduced bond current for the four-membered ring remains net paratropic $\left(J=\frac{1}{4 \sqrt{5}}-1 / 4 ;-0.138\right)$ and corresponds to half the current of a benzene ring which has $9 / 4$ times the area, and again represents the pattern of nitrogen-centred circulations in a more detailed description.

Generalisation for $4 n$ and $(4 n+2) \pi B N$ heterocycles: The results for the four-, six- and eight-membered cycles are generalisations for the $4 n$ and $(4 n+2) \pi \mathrm{BN}$ heterocycles, with separate mechanisms, but equivalent results for the ring current in both cases. Consider an $[N]$-carbocycle, where $N$ is even. As $\eta$ increases from 0 , each bonding $(b)$ /antibonding (a) molecular-orbital pair of the parent bipartite carbocycle, with angular momenta $\Lambda_{b}$ and $\Lambda_{a}=N / 2-\Lambda_{b}$, which correspond to equal but opposite eigenvalues, mix to form a bonding/antibonding pair in the $[N]$-centre $\mathrm{BN}$ heterocycle with energies $x_{b}=\sqrt{4 \cos ^{2} \tau_{A b}+\eta^{2}}$ and $x_{a}=-\sqrt{4 \cos ^{2} \tau_{A a}+\eta^{2}}$, where $\tau_{\Lambda}=2 \pi \Lambda / N$, so that $x_{b}=-x_{a}$. The rotation describing the mixing is parametrised by the angle $\omega_{\Lambda}=1 / 2 \tan ^{-1}(\eta /$ $2 \cos \tau_{\Lambda}$ ), where $0 \leq \omega_{\Lambda} \leq \pi / 4$.

When $N=4 n+2, \Lambda_{\mathrm{HOMO}}=n$ and $\Lambda_{\mathrm{LUMO}}=n+1$, and the mixing angle $\omega_{\Lambda}$ is maximal for a given value of $\eta$. As we have seen, this leads to a sufficiently large value of $\eta$ to disrupt the global ring current and eventual localisation. When $N=4 n, \Lambda_{\text {HOMO }}=\Lambda_{\mathrm{LUMO}}=n$, the original HOMO and LUMO carbocycle eigenfunctions remain unmixed. Disruption of the global paratropic ring current is caused by the widening of the HOMO-LUMO gap, triggering cancellation of the rotational HOMO-LUMO and translational HOMO-1LUMO contributions.

Ab initio current-density maps in azabora-heterocycles: To obtain ab initio data on the currents in $\mathrm{BN}$ analogues of the $4 n$ and $4 n+2$ carbocycles, calculations were performed on $\mathrm{B}_{2} \mathrm{~N}_{2} \mathrm{H}_{4}, \mathrm{~B}_{3} \mathrm{~N}_{3} \mathrm{H}_{6}$ and $\mathrm{B}_{4} \mathrm{~N}_{4} \mathrm{H}_{8}$. The geometry of each molecule was optimised at the restricted Hartree-Fock (RHF) level with the $6-31 \mathrm{G}^{* *}$ basis set and currents were calculated by using the ipsocentric approach at the coupled Hartree-Fock (CHF) level with the same basis set. At this level of theory, $\mathrm{B}_{3} \mathrm{~N}_{3} \mathrm{H}_{6}$ and $\mathrm{B}_{4} \mathrm{~N}_{4} \mathrm{H}_{8}$ have planar structures with $D_{3 h}$ and $D_{4 h}$ symmetries, respectively, whereas the planar structure of $\mathrm{B}_{2} \mathrm{~N}_{2} \mathrm{H}_{4}$ with $D_{2 h}$ symmetry is a transition state (imaginary frequency $116 i \mathrm{~cm}^{-1}$ ) that leads to a shallow $C_{2 v}$ butterfly optimum, hinged at the $\mathrm{BB}$ diagonal, with a dihedral angle of $167^{\circ}$. Current-density maps were computed for both the constrained planar and the fully optimised structures of $\mathrm{B}_{2} \mathrm{~N}_{2} \mathrm{H}_{4}$. An alternative geometry for $\mathrm{B}_{4} \mathrm{~N}_{4} \mathrm{H}_{8}$ is based on the occupation of the corners of a cube $;^{[25 a]}$ this compact structure is slightly preferred to the planar form, as determined with small basis sets, ${ }^{[25 \mathrm{~b}]}$ but lies $302 \mathrm{~kJ} \mathrm{~mol}^{-1}$ above it when calculated at the $6-31 \mathrm{G}^{* *}$ level. All three planar molecules have uniform $\mathrm{BN}$ distances $(R=1.4432$, $1.4258,1.4254 \AA$, respectively).

These geometries are in general agreement with available experimental and theoretical data. The nonplanarity of $\mathrm{B}_{2} \mathrm{~N}_{2} \mathrm{H}_{4}$ has been noted in previous ab initio calculations, ${ }^{[26]}$ which gave a $\mathrm{BN}$ distance of $1.457 \AA$, and although the parent molecule itself has not been synthesised, X-ray structures of five substituted molecules ${ }^{[14 \mathrm{~b}]}$ are known with mean BN distances varying between 1.430 and $1.486 \AA$, and all are planar apart from the severely hindered tetra-tert-butyl derivative. Borazine has been the subject of many ab initio calculations. $^{[27]}$ The RHF//6-31G**-calculated BN distance of $1.4258 \AA$ in borazine compares with $1.4355 \pm 0.0021 \AA$ (gas electron diffraction $\left.{ }^{[28 \mathrm{a}, \mathrm{b}]}\right)$ and $1.429 \AA\left(\mathrm{X}-\right.$ ray $\left.^{[28 \mathrm{c}]}\right)$. No experimental data are available for the parent $\mathrm{B}_{4} \mathrm{~N}_{4} \mathrm{H}_{4}$, but experimental and theoretical structural data on its derivatives have been reviewed by Gilbert and Gailbreath. ${ }^{[26]}$ Calculations at different levels give a planar (B3LYP/6-31G+*) or near-planar (MP2/6-31G+*) structure for $\mathrm{B}_{4} \mathrm{~N}_{4} \mathrm{H}_{8}$ with a $\mathrm{BN}$ length of $1.436 \AA$, intermediate between single and double bonds. Equivalent calculations on the permethylated mono- 

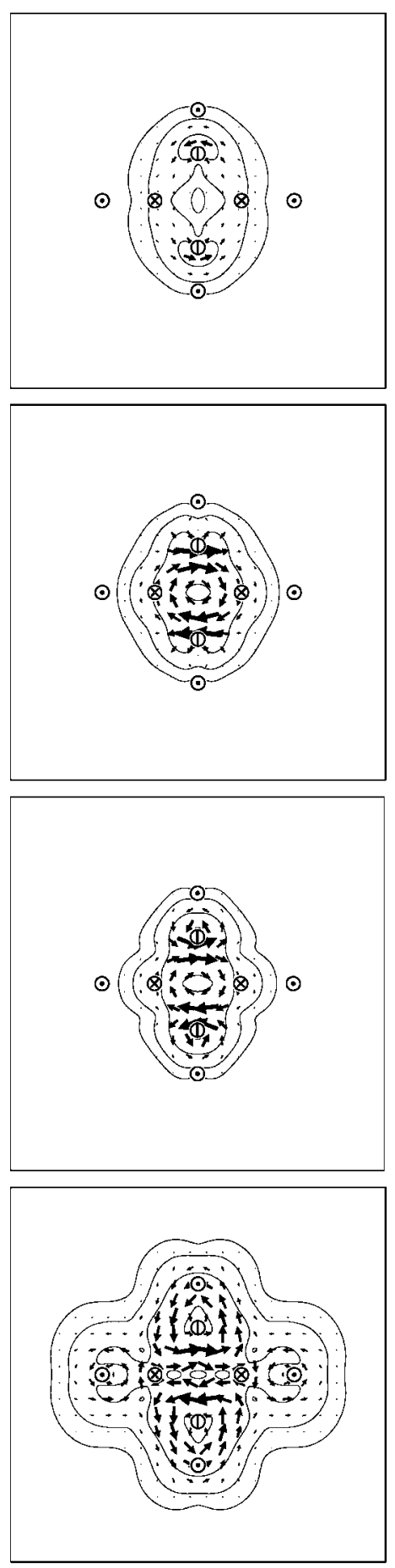
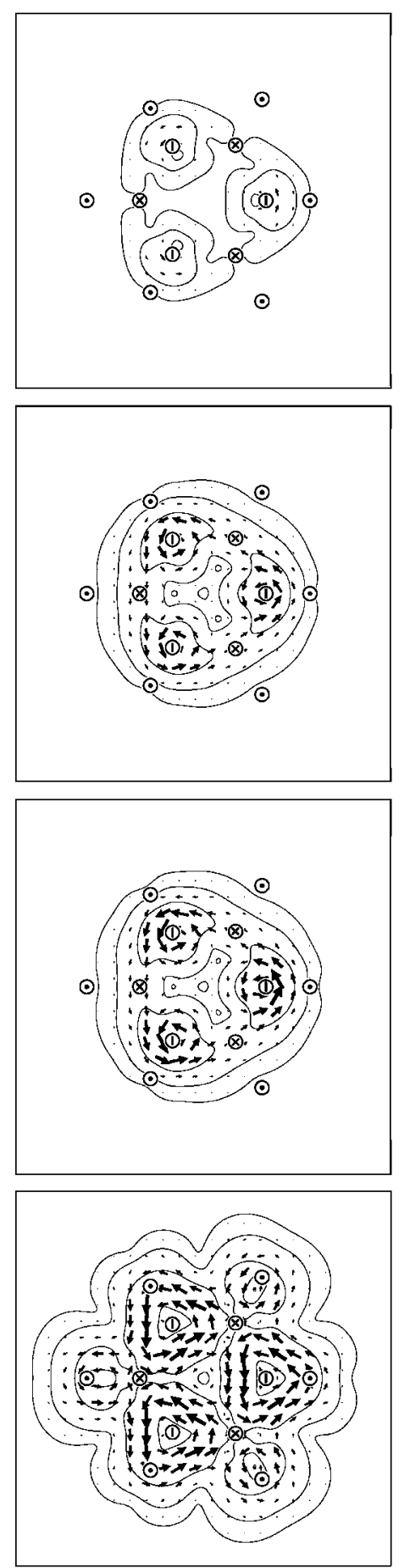
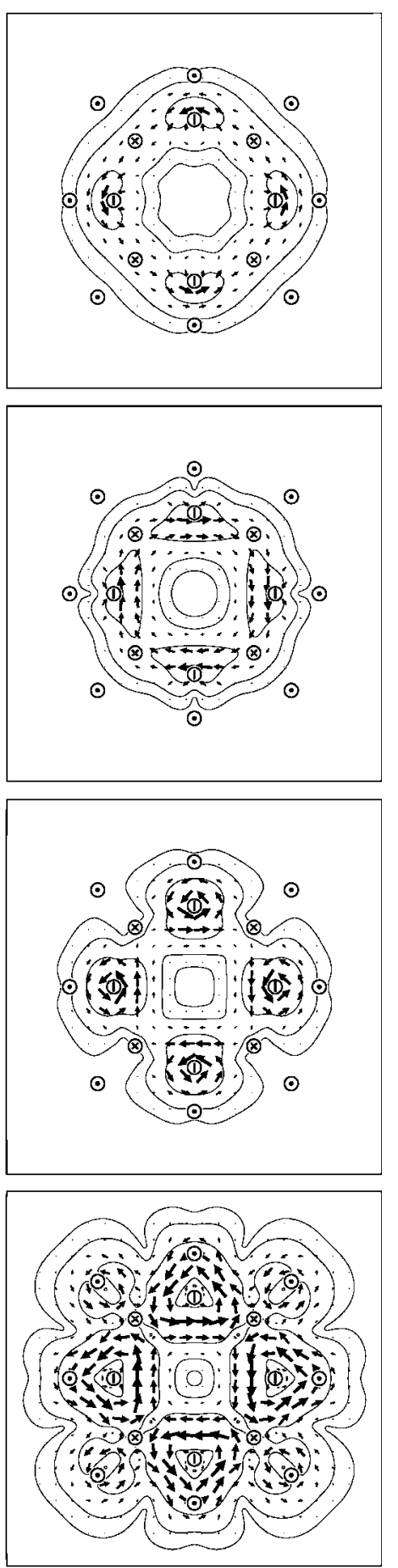

Figure 4. Current density induced by a perpendicular external magnetic field in the molecules $\mathrm{B}_{2} \mathrm{~N}_{2} \mathrm{H}_{4}\left(\mathbf{1}\right.$, planar), $\mathrm{B}_{3} \mathrm{~N}_{3} \mathrm{H}_{6}(\mathbf{2}), \mathrm{B}_{4} \mathrm{~N}_{4} \mathrm{H}_{8}(\mathbf{3})($ left to right), partitioned as contributions from (top to bottom) the HOMO-1, HOMO, $\pi$ and $\sigma$ orbitals, calculated by the RHF/6-31G** ipsocentric approach. Where the HOMO-1 and HOMO are degenerate, the map shows the summed contribution of the pair. Contributions are plotted at one bohr above the molecular plane. Anticlockwise circulations are diatropic, clockwise circulations paratropic. Nitrogen, boron and hydrogen centres are denoted by circles containing a bar, cross or dot, respectively.

cycle show it to adopt a tub structure. ${ }^{[26]} \mathrm{X}$-ray structures for derivatives with bulky substituents are tub-like with alternating $\mathrm{BN}$ bonds as in, for example, $(t \mathrm{BuN})_{4}(\mathrm{MeB})_{4} \cdot{ }^{\left[{ }^{[2]}\right.}$ Solution ${ }^{1} \mathrm{H}$ NMR spectra of $\mathrm{B}_{4} \mathrm{~N}_{4}\left(\mathrm{CH}_{2} \mathrm{R}\right)_{4}(\mathrm{Me})_{4}$ show a nonplanar eight-membered ring. ${ }^{[30]}$
The current densities induced in (planar) $\mathrm{B}_{2} \mathrm{~N}_{2} \mathrm{H}_{4}, \mathrm{~B}_{3} \mathrm{~N}_{3} \mathrm{H}_{6}$ and $\mathrm{B}_{4} \mathrm{~N}_{4} \mathrm{H}_{8}$ by a perpendicular magnetic field are plotted in Figure 4. For each molecule, the maps show HOMO, HOMO -1 and the total $\pi$ and $\sigma$ contributions to the induced current density plotted in a plane one bohr above 
that of the nuclei. As Figure 4 shows, all three systems, in contrast to the carbocycles, have multicentre patterns of local diatropic $\pi$ circulations centred on the nitrogen atoms. In $\mathrm{B}_{3} \mathrm{~N}_{3} \mathrm{H}_{6}$, the nitrogen-centred $\pi$ circulations arise entirely from the four electrons of the doubly degenerate HOMO: the total $\pi$ and HOMO maps are visually indistinguishable, and the contribution of the HOMO-1 is negligible. In $\mathrm{B}_{2} \mathrm{~N}_{2} \mathrm{H}_{4}$ and $\mathrm{B}_{4} \mathrm{~N}_{4} \mathrm{H}_{8}$, the nitrogen-centred $\pi$ circulations arise from a combination of the inner paratropic HOMO and the outer diatropic HOMO-1 currents. As the localised $\sigma$ and $\pi$ currents rotate in the same sense, they are reinforced in all three molecules. Maps of total current density (not shown) plotted for the puckered optimum geometry of $\mathrm{B}_{2} \mathrm{~N}_{2} \mathrm{H}_{4}$ at a height of $1 \mathrm{a}_{0}$ above the nitrogen atoms and parallel to the median nuclear plane show the same general features: the localised nature of the current density is not critically dependent on planarity.

Localised orbital analysis: Further analysis using PipekMezey localisation of the canonical molecular orbitals ${ }^{[31]}$ demonstrates the strong generic similarity of all three systems. The localised orbitals of $\mathrm{B}_{N / 2} \mathrm{~N}_{N / 2} \mathrm{H}_{N}$ are $N$ core 1 s orbitals on the heavy atoms, $N / 2 \mathrm{BN}$ localised $\sigma$-bonding orbitals, N/2 NH and N/2 BH localised $\sigma$-bonding orbitals and $N / 2 \pi$ orbitals that are essentially nitrogen-localised $\pi$ lone pairs. In this analysis nitrogen-centred $\pi$ currents arise naturally as circulations in the lone pairs. The $\sigma$ currents shown in Figure 4 for $\mathrm{B}_{2} \mathrm{~N}_{2} \mathrm{H}_{4}, \mathrm{~B}_{3} \mathrm{~N}_{3} \mathrm{H}_{6}$ and $\mathrm{B}_{4} \mathrm{~N}_{4} \mathrm{H}_{8}$ have nodes at the boron sites. In the localised-orbital picture, the prominent "triangular" diatropic circulations around the nitrogen atoms arise from the three bonds meeting at nitrogen, each deltoid constituting a six-electron diatropic circulation. In the carbocycles, the $\sigma$ currents are more uniformly distributed over all the heavy atoms, but are still spatially localised. ${ }^{[32]}$ The main difference in the magnetic response between carbocycles and azabora-heterocycles is that the $\pi$ ring currents of one class are absent in the other. This distinction is clear from the computed current-density maps, which are fully compatible with the qualitative analysis based on symmetry and electronegativity. One and the same ipsocentric molecular-orbital picture explains both the current in the carbocycle and the lack of current in the heterocycle.

Valence-bond calculations on azabora-heterocycles: As the localisation of density on electronegative nitrogen centres is a key factor in the simple Hückel model of ring-current quenching, it is important to verify this qualitative difference between carbocyclic and azabora-heterocyclic systems by using more sophisticated theoretical methods. Accordingly, ab initio valence-bond configuration-interaction (VBCI) calculations, in which many structures are used, and valence-bond self-consistent field (VBSCF) ${ }^{[33]}$ calculations, in which both orbitals and structure coefficients are optimised, were performed on planar $\mathrm{B}_{2} \mathrm{~N}_{2} \mathrm{H}_{4}$ (1), $\mathrm{B}_{3} \mathrm{~N}_{3} \mathrm{H}_{6}$ (2) and $\mathrm{B}_{4} \mathrm{~N}_{4} \mathrm{H}_{8}$ (3) with the 6-31G** basis set. All were carried out by using TURTLE, ${ }^{[34]}$ as implemented in the GAMESS-UK package. ${ }^{[35]}$
The wavefunction in the valence-bond calculations consists of a Hartree-Fock $\sigma$ core in structures with singly occupied $\pi$ orbitals. From a chemical point of view, these different structures can be seen as different bonding arrangements. All possible structures that can be generated by using the occupied atomic orbitals were used in the VBCI wavefunction, including chemically irrelevant ones. For $\mathrm{B}_{2} \mathrm{~N}_{2} \mathrm{H}_{4}$ there are 20 possible structures which arise from the 20 ways of distributing four electrons over four $\mathrm{p}$ orbitals to give a singlet. For $\mathrm{B}_{3} \mathrm{~N}_{3} \mathrm{H}_{6} 175$ structures are possible, and for $\mathrm{B}_{4} \mathrm{~N}_{4} \mathrm{H}_{8}$ there are 1764. A VBCI calculation will show which bonding arrangements are preferred.

However, the VBCI wavefunction, owing to its fixed atomic orbital basis set, does not present a compact description of the wavefunction as orbital optimisation and correlation effects are intermixed. Therefore the most important structures in the VBCI calculations were used to construct a VBSCF wavefunction without imposing restrictions on the orbitals. From the coefficients in the VBCI wavefunctions the weights, ${ }^{[36]}$ and thus the importance of each structure, may be derived. For 1-3, the most important structure types are shown in Figure 5 with their respective weights. From these weights it appears that several structures contribute to the total wavefunction.

Subsequently, for $\mathrm{B}_{2} \mathrm{~N}_{2} \mathrm{H}_{4}$, the sets of structures of each kind were separately optimised in VBSCF calculations with-

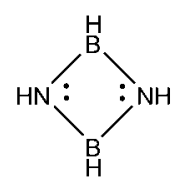

$1 \mathrm{a}$

$(1 \times 40.4 \%)$<smiles>B1NBNBN1</smiles>

$2 a$

$(1 \times 17.9 \%)$

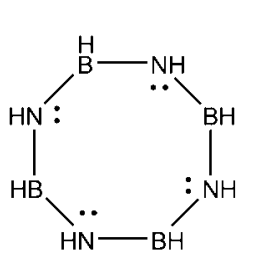

$3 a$

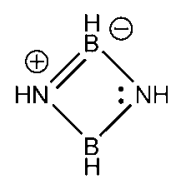

$1 \mathrm{~b}$

$(4 \times 12.2 \%)$<smiles>B1NB2NBNB2N1</smiles>

$2 b$

$(6 \times 6.4 \%)$<smiles>[B]1NBNBNBN1</smiles>

$3 b$

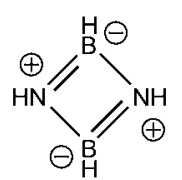

$1 c$

$(2 \times 4.2 \%)$

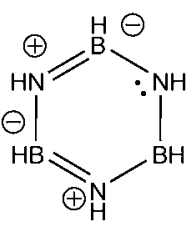

2c

$(6 \times 3.5 \%)$
$(1 \times 18.0 \%)$

$(8 \times 8.8 \%)$

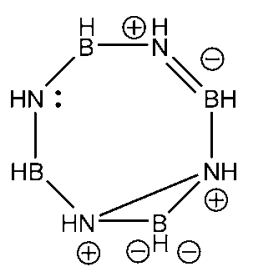

$3 \mathrm{c}$

$(8 \times 1.3 \%)$
Figure 5. The most significant symmetry-distinct VBCI structures of $\mathrm{B}_{2} \mathrm{~N}_{2} \mathrm{H}_{4}(\mathbf{1}), \mathrm{B}_{3} \mathrm{~N}_{3} \mathrm{H}_{6}(2)$ and $\mathrm{B}_{4} \mathrm{~N}_{4} \mathrm{H}_{8}(3)$ with the total weight of the combined set in parentheses. 
out imposing any restrictions on the orbitals. The resulting energies of the first $\left(\mathbf{1} \mathbf{a}:-160.722209 E_{\mathrm{h}}\right)$ and the second (1) : $-160.721245 E_{\mathrm{h}}$ ) were very similar, with the first structure, having two different orbitals on each nitrogen atom (see Figure 6), slightly more favourable.
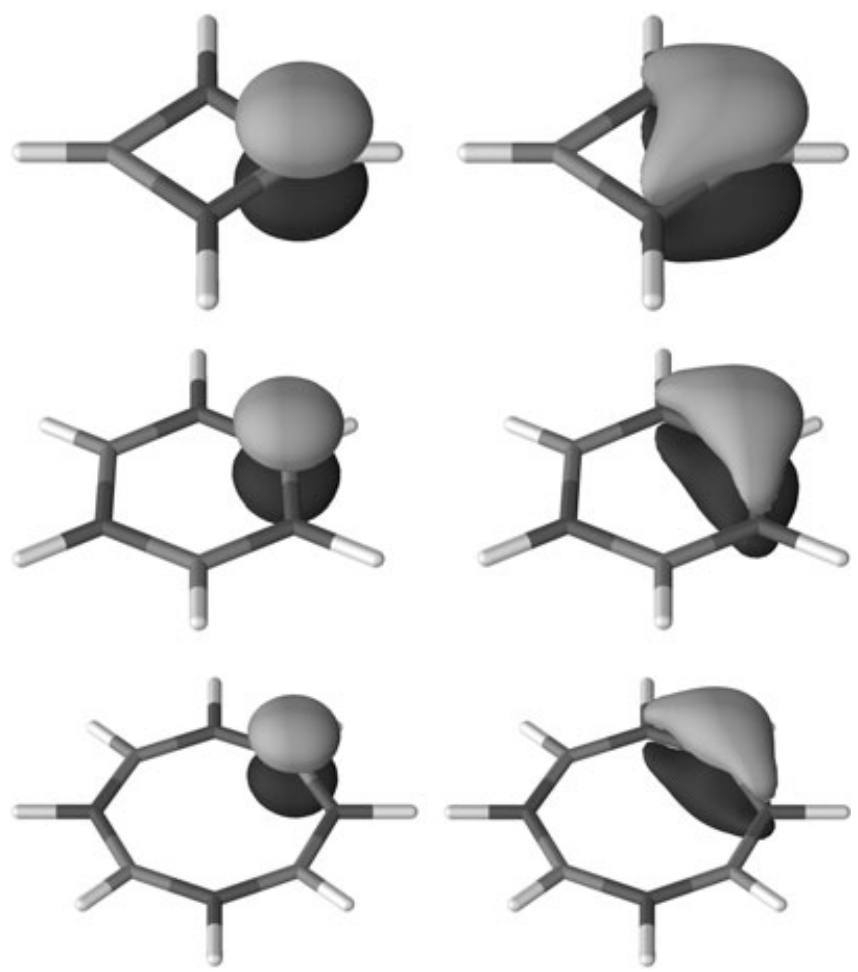

Figure 6. The singly occupied p orbitals of $\mathrm{B}_{2} \mathrm{~N}_{2} \mathrm{H}_{4}(\mathbf{1}), \mathrm{B}_{3} \mathrm{~N}_{3} \mathrm{H}_{6}(2)$ and $\mathrm{B}_{4} \mathrm{~N}_{4} \mathrm{H}_{8}(3)$, respectively, which correspond to the structures $1 \mathbf{a}, \mathbf{2} \mathbf{a}$ and 3a (see Figure 5). Orbitals from the left and right of the picture together form the correlated lone pair on the nitrogen atom.

A VBCI calculation was then performed on the first two sets of structures $(\mathbf{1} \mathbf{a}+\mathbf{1} \mathbf{b})$, each with their own optimised orbitals. This calculation gave the same energy as the single structure VBSCF calculation (1a), whereas the overlap between set one (1a) and set two (1) was over $99.9 \%$. This means that the VBSCF calculations converge to the same wavefunction, which is most aptly described as nitrogen atoms, each with a correlated pair of $\pi$ electrons. The conclusion is that there is no resonance energy and thus no resonance. ${ }^{[37]}$ Similar results were obtained for $\mathrm{B}_{3} \mathrm{~N}_{3} \mathrm{H}_{6}(2)^{[37]}$ and $\mathrm{B}_{4} \mathrm{~N}_{4} \mathrm{H}_{8}(3)$.

These calculations show the same tendency as both the Hückel and the ring current calculations, that is, localisation of electrons on the nitrogen atoms. In fact, the valence-bond results show that the ground states of $\mathrm{B}_{2} \mathrm{~N}_{2} \mathrm{H}_{4}(\mathbf{1}), \mathrm{B}_{3} \mathrm{~N}_{3} \mathrm{H}_{6}$ (2) and $\mathrm{B}_{4} \mathrm{~N}_{4} \mathrm{H}_{8}(\mathbf{3})$ correspond to the structures $\mathbf{1 a}, \mathbf{2} \mathbf{a}^{[37]}$ and $\mathbf{3} \mathbf{a}$, respectively.

\section{Conclusions}

Given the success of the simple one-parameter model in pointing out qualitative differences in current patterns between carbo- and azabora-cycles, it is natural to ask about its predictions for other systems, for example, $\mathrm{X}_{N / 2} \mathrm{Y}_{N / 2} \mathrm{H}_{N}-$ where does the borderline between "aromatic" and "nonaromatic" occur in this picture? The crudest form of the model shows a change from global circulation to localised currents by a strong reduction in the magnitude of ring current from $\eta=0$ to $\eta=1$; this variation is sigmoidal (Figure 7)

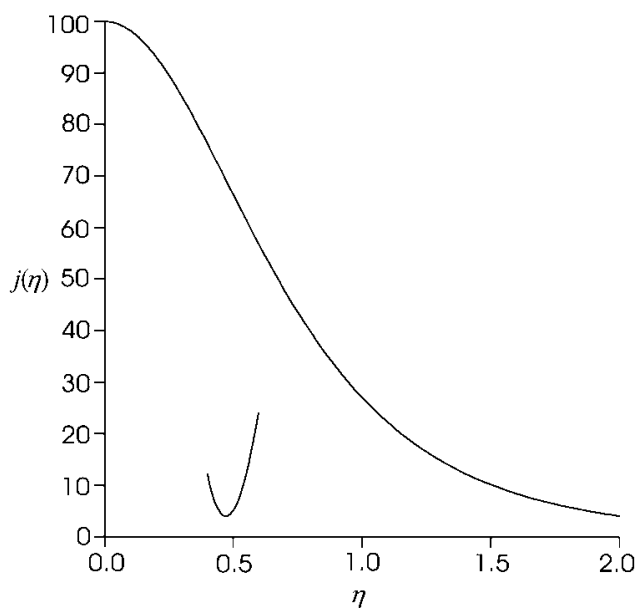

Figure 7. Variation of ring current, $j$, with electronegativity parameter, $\eta$, in the Hückel-London treatment of the $\mathrm{X}_{3} \mathrm{Y}_{3}$ cycle. $j(\eta)$ is the ring current for a regular hexagonal cycle with alternating coulomb parameters $\alpha \pm \eta \beta$ as a percentage of the current for the same cycle with $\eta=0$. The inset curve (not to scale) shows the variation of the first derivative $\operatorname{d} j(\eta) /$ $\mathrm{d} \eta$ near $\eta=0.5$.

with a point of inflexion, where current will be most sensitive to variation in $\eta$, at $\eta \approx 0.5$, where $\eta$ represents the deviation from the average electronegativity, that is, $\left|\alpha_{X}-\alpha_{Y}\right| 2 \beta$. A rule of thumb is that $\alpha_{\mathrm{X}}$ varies with the difference in electronegativity between $\mathrm{X}$ and $\mathrm{C}:\left(\alpha_{\mathrm{X}}-\alpha_{\mathrm{C}}\right) / \beta \approx \chi_{\mathrm{X}}-\chi_{\mathrm{C}}$ for $\mathrm{X}$ as a one-electron donor and $\left(\alpha_{\mathrm{X}}-\alpha_{\mathrm{C}}\right) / \beta \approx 1+\chi_{\mathrm{X}}-\chi_{\mathrm{C}}$ as a twoelectron donor ${ }^{[22 \mathrm{a}]}$ ). Inflexion at $\eta \approx 0.5$ is consistent with indications for $\mathrm{X}_{3} \mathrm{Y}_{3} 6 \pi$ systems from ring-current maps ${ }^{[2]}$ and NICS calculations: ${ }^{[27 c]}$ systems in which the electronegativity/coulomb-parameter difference between $\mathrm{X}$ and $\mathrm{Y}$ is 1 or more on the Pauling scale lack ring currents $\left(\mathrm{B}_{3} \mathrm{O}_{3} \mathrm{H}_{3},{ }^{[2,27 \mathrm{c}]}\right.$ $\mathrm{B}_{3} \mathrm{~N}_{3} \mathrm{H}_{6},{ }^{[2,27 \mathrm{c}]} \quad \mathrm{Al}_{3} \mathrm{~N}_{3} \mathrm{H}_{6}{ }^{[27 \mathrm{c}]}$ and $\left.\mathrm{Al}_{3} \mathrm{P}_{3} \mathrm{H}_{6}{ }^{[27 \mathrm{c}]}\right)$, whereas those with differences of half a unit or less may $\left(\mathrm{C}_{3} \mathrm{~N}_{3} \mathrm{H}_{3}{ }^{[2,27 \mathrm{c}]}\right.$ and $\left.\mathrm{B}_{3} \mathrm{P}_{3} \mathrm{H}_{6}{ }^{[27 c]}\right)$ or may not $\left(\mathrm{B}_{3} \mathrm{~S}_{3} \mathrm{H}_{3}{ }^{[27 \mathrm{c}]}\right)$ show global currents. This uniform trend lends support to the expectation that orbital-based models should be able to account for the subtleties of ring current in the range of aromatic, nonaromatic and antiaromatic heterocycles, and is a strong argument for using methods in which the orbital contributions themselves have a clear physical basis. 


\section{Acknowledgements}

L.W.J. acknowledges financial support from the Royal Society of Chemistry's Journals Grants for International Authors Programme (0301433). R.W.A.H. acknowledges financial support from the Netherlands Organisation for Scientific Research (NWO), grant 700.53.401. P.W.F, A.R. and A.S. thank the EU for financial support from the Framework $\mathrm{V}$ programme (RTN Contract HPRN-CT-2002-00136 "WONDERFULL"), C.D. thanks the Royal Society for a University Research Fellowship and P.W.F. for a Royal Society/Wolfson Research Merit Award.

[1] R. C. Haddon, J. Am. Chem. Soc. 1979, 101, 1722-1728.

[2] P. W. Fowler, E. Steiner, J. Phys. Chem. A 1997, 101, 1409-1413.

[3] E. Steiner, P. W. Fowler, J. Phys. Chem. A 2001, 105, 9553-9562.

[4] E. Steiner, P. W. Fowler, Chem. Commun. 2001, 2220-2221.

[5] E. Steiner, P. W. Fowler, R. W. A. Havenith, J. Phys. Chem. A 2002, 106, 7048-7056.

[6] F. London, J. Phys. Radium 1937, 8, 397-409.

[7] L. Pauling, J. Chem. Phys. 1936, 4, 673-677.

[8] J. A. Pople, J. Chem. Phys. 1956, 24, 1111..

[9] P. von R. Schleyer, C. Maerker, A. Dransfeld, H. Jiao, N. J. R. van Eikema Hommes, J. Am. Chem. Soc. 1996, 118, 6317-6318.

[10] H. C. Longuet-Higgins in Aromaticity, Special Publication No. 21, The Chemical Society, London, 1967, 109-110.

[11] For recent summaries of the history of the discussion on the putative aromatic character of borazine, see, for example: a) M. A. M. Forgeron, D. L. Bryce, R. E. Wasylishen, R. Rosler, J. Phys. Chem. A 2003, 107, 726-735; b) B. Kiran, A. K. Phukan, E. D. Jemmis, Inorg. Chem. 2001, 40, 3615-3618.

[12] G. A. Anderson, J. J. Lagowski, Inorg. Chem. 1975, 14, 1845-1848.

[13] A. J. Bridgeman, Polyhedron 1998, 17, 2279-2288.

[14] For references to the chemistry, and to discussions on the possible aromaticity of these molecules, see: a) P. Paetzold, Phosphorus, Sulfur Silicon Relat. Elem. 1994, 93, 39-50; b) E. von Steuber, G. Elter, M. Noltemeyer, H. G. Schmidt, A. Meller, Organometallics 2000, 19, 5083-5091.

[15] R. C. Haddon, Pure Appl. Chem. 1982, 54, 1129-1142.

[16] N. C. Baird, M. A. Whitehead, Can. J. Chem. 1967, 45, 2059-2070.

[17] T. A. Keith, R. F. W. Bader, Chem. Phys. Lett. 1993, 210, 223-231.

[18] S. Coriani, P. Lazzeretti, M. Malagoli, R. Zanasi, Theor. Chim. Acta 1994, 89, 181-192.

[19] A. Soncini, P. W. Fowler, L. W. Jenneskens, Phys. Chem. Chem. Phys. 2004, 6, 277-284.

[20] a) A. Matsuura, K. Komatsu, J. Am. Chem. Soc. 2001, 123, 17681769 ; b) P. W. Fowler, R. W. A. Havenith, L. W. Jenneskens, A. Soncini, E. Steiner, Angew. Chem. 2002, 114, 1628-1630; Angew. Chem. Int. Ed. 2002, 41, 1558-1560.

[21] R. Daudel, R Lefebvre, C. Moser, Quantum Chemistry, Methods and Applications, Wiley-Interscience, New York, 1959, p. 449.
[22] a) A. Streitwieser Jr., Molecular Orbital Theory for Organic Chemists, Wiley, New York, 1961; b) N. C. Baird, M. A. Whitehead, Can. J. Chem. 1966, 44, 1933-1943.

[23] R. McWeeny, Mol. Phys. 1958, 1, 311-321.

[24] P. W. Fowler, E. Steiner, Chem. Phys. Lett. 2002, 364, 259-266.

[25] a) H. S. Turner, R. J. Warne, Proc. Chem. Soc. London 1962, 69-70; b) R. M. Minyaev, J. Struct. Chem. 2000, 41, 1-10.

[26] T. M. Gilbert, B. D. Gailbreath, Organometallics 2001, 20, 47274733 , and references therein.

[27] For example, see: a) E. F. Archibong, A. J. Thakkar, Mol. Phys. 1994, 81, 557-567; b) F. Ramondo, G. Portalone, M. Colapietro, L. Bencivenni, J. Mol. Struct. 1993, 302, 85-99; c) P. von R. Schleyer, H. Jiao, N. J. R. van Eikema Hommes, V. G. Malkin, O. L. Malkina, J. Am. Chem. Soc. 1997, 119, 12669-12670; d) E. Steiner, P. W. Fowler, R. W. A. Havenith, J. Phys. Chem. A 2002, 106, 7048-7056; e) E. D. Jemmis, B. Kiran, Inorg. Chem. 1998, 37, 2110-2116; f) S. Kawahara, S. Tsuzuki, T. Uchimaru, J. Chem. Phys. 2003, 119, 10081-10087.

[28] a) W. Harshbarger, G. H. Lee, R. F. Porter, S. H. Bauer, J. Am Chem. Soc. 1969, 91, 551-555; b) W. Harshbarger, G. H. Lee, R. F. Porter, S. H. Bauer, Inorg. Chem. 1969, 8, 1683-1689; c) R. Böse, A. H. Maulitz, P. Stellberg, Chem. Ber. 1994, 127, 1887-1889.

[29] T. Franz, E. Hanecker, H. Nöth, W. Stöcker, W. Storch, G. Winter, Chem. Ber. 1986, 119, 900-916.

[30] B. Thiele, P. Schreyer, U. Englert, P. Paetzold, R. Böse, B. Wrackmeyer, Chem. Ber. 1991, 124, 2209-2216.

[31] J. Pipek, P. G. Mezey, J. Chem. Phys. 1989, 90, 4916-4926.

[32] E. Steiner, P. W. Fowler, Phys. Chem. Chem. Phys. 2004, 6, 261-272.

[33] J. H. van Lenthe, G. G. Balint-Kurti, J. Chem. Phys. 1983, 78, 56995713.

[34] TURTLE, an ab initio VB/VBSCF program, J. Verbeek, J. H. Langenberg, C. P. Byrman, F. Dijkstra, J. J. Engelberts, J. H. van Lenthe, 1988-2004.

[35] GAMESS-UK (2004) is a package of ab initio programs written by M. F. Guest, J. H. van Lenthe, J. Kendrick, K. Schoffel and P. Sherwood, with contributions from R. D. Amos, R. J. Buenker, H. J. J. van Dam, M. Dupuis, N. C. Handy, I. H. Hillier, P. J. Knowles, V. Bonacic-Koutecky, W. von Niessen, R. J. Harrison, A. P. Rendell, V. R. Saunders, A. J. Stone, D. J. Tozer and A. H. de Vries. The package is derived from the original GAMESS code: M. Dupuis, D. Spangler, J. Wendoloski, NRCC Software Catalog, Vol. 1, Program No. QG01 (GAMESS), 1980

[36] B. H. Chirgwuin , C. A. Coulson, Proc. R. Soc. London 1950, A201, 196-209.

[37] D. L. Cooper, S. C. Wright, J. Gerratt, P. A. Hyams, J. Chem. Soc., Perkin Trans. 2 1989, 6, 719-724. 